\title{
Neurological Symptoms and Evaluation of Cerebral Blood Flow Changes with Synthetic Cannabinoids Use
}

\author{
Onur Karakayali1, Uygar Utku2, Serkan Yilmaz³ and Nevzat Uzuner4 \\ 1Department of Emergency Medicine, Human Health University, Derince Training and Research Hospital, Kocaeli, Turkey \\ ${ }^{2}$ Department of Neurology, Marash State Hospital, Kahramanmaras, Turkey \\ ${ }^{3}$ Department of Emergency, Faculty of Medicine, Kocaeli University, Kocaeli, Turkey \\ ${ }^{4}$ Department of Neurology, Faculty of Medicine, Osmangazi, Eskisehir, Turkey
}

\begin{abstract}
Objective: To describe the cerebral haemodynamic changes that occur in patients presenting to the emergency department (ED) after synthetic cannabinoid (SC) use in order to clarify the pathophysiology of neurologic adverse effects of SCs.

Study Design: Observational study.

Place and Duration of Study: Emergency Department of Kocaeli Derince Training and Research Hospital, Turkey, from June 2017 to January 2018.

Methodology: Patients in whom tetrahydrocannabinol was detected in the urinalysis due to SC use were included in the study. Healthy controls were also included. All the participants were evaluated using transcranial Doppler ultrasonography (TCD). The peak systolic flow rate, mean flow rate, pulsatility index and resistivity index of the right and the left anterior carotid arteries, middle cerebral arteries and posterior cerebral arteries were measured.

Results: A total of 74 patients were included in this study - 38 patients in the SC group and 36 patients in the control group. The mean age of all of the subjects was $29.6 \pm 9.1$ years, whereas, $91.9 \%$ of them were males. In the synthetic cannabinoids group, statistically significant decrease in the cerebral blood flow peak systolic and mean values as well as increases in the pulsatility index; and resistivity index were found via TCD when compared to the control group.

Conclusion: Cerebral blood flow and resistance changes due to SC use are likely to play a role in the main pathogenesis of the neurological symptoms, increasing the frequency of ischemic or haemorrhagic strokes.
\end{abstract}

Key Words: Cerebral blood flow, Neuronal damage, Synthetic cannabinoids, Transcranial Doppler ultrasound.

How to cite this article: Karakayali O, Utku U, Yilmaz S, Uzuner N. Neurological symptoms and evaluation of cerebral blood flow changes with synthetic cannabinoids use. J Coll Physicians Surg Pak 2019; 29(12):1183-1188.

\section{INTRODUCTION}

Synthetic cannabinoid (SC), make-up a family of chemical compounds that are similar to delta-9tetrahydrocannabinol (THC), is an important public health problem. ${ }^{1}$ There is little information about the structural and functional neurologic damage due to the fact that the use of SCs cannot be established clearly by the active substances when compared to narcotic drugs. ${ }^{2}$ Case series and retrospective studies have shown that there are conflicting results regarding the pathophysiology of acute neurological complications but prospective control group studies are limited.3,4 Neurological involvement due to SC use has been reported in many studies, and whether this effect is due to active metabolites of SCs or changes in cerebral blood flow and cerebral blood flow changes have not been described in the literature.

Correspondence to: Dr. Onur Karakayali, Department of Emergency Medicine, Human Health University, Derince Training and Research Hospital, Kocaeli, Turkey

E-mail:dr_onurkarakayali@hotmail.com

Received: May 07, 2019; Revised: August 07, 2019;

Accepted: September 30, 2019
Conventionally, no stroke relationships to the use of marijuana or SCs have been demonstrated; however, cases of ischemic or haemorrhagic strokes have been described in emergency services in case series in the literature.5,6 Unfortunately, the stroke pathophysiology has not been elucidated in these similar case series due to the limited number of prospective studies.

In limited studies, it has been suggested that changes in cerebral blood flow may be the cause of neurologic changes due to SC use. ${ }^{7}$ Transcranial Doppler (TCD) ultrasonography (USG) is the most commonly used imaging modality for detecting abnormal haemodynamic changes in cerebral arteries. TCD USG is widely used in the determination of stroke risks in different patient groups. In addition, it can be used effectively in vasospasm monitoring, such as in cases of subarachnoid haemorrhages, the detection of intracranial stenoocclusive diseases and cerebral thrombosis monitoring. ${ }^{8}$

This study was conducted to describe the cerebral haemodynamic changes in patients presenting to the ED after SC use in order to clarify the pathophysiology and possible neuronal damage due to the adverse neurological effects of SCs. 


\section{METHODOLOGY}

This observational study was carried out from June 2017 to January 2018 at Emergency Department (ED), Kocaeli Derince Training and Research Hospital, Turkey as a single centre. This study included patients presenting with adverse symptoms due to SC use. Approval for this study was obtained from the local Ethics Committee (KU GOKAEK, 2016/317). It was conducted in accordance with the principles of the World Helsinki Declaration and Guidelines for Good Clinical Practice. All of the participants were informed before being included in the study, and consents were obtained from all the participants or their relatives.

Patients over the age of 18 years, who were admitted to ED with clinical signs and symptoms related to SC, who were suspected of SC use with regard to the medical history and physical examination findings, and those with SCs detected in their urinalyses were included. The exclusion criteria were patients under 18 years of age, with no THC detected in their urinalyses, who used other substances including ethanol detected in their blood analyses, unstable patients at the time of presentation, known malignant central nervous system neoplasms, patients with histories of ischemic or haemorrhagic cerebrovascular diseases or infections, trauma, patients with closed temporal windows for TCD USG measurements, and patients who did not give their consent to participate. The control group who were over 18 years of age and healthy with exclusion criteria, no history of previous substance abuse or alcohol use or smoking or tobacco consumption, and provided consent to participate were included.

All patients included in this study were evaluated by the emergency medicine specialist in the ED. The demographic data, substance use history, presence of additional trauma and complaints at presentation were recorded. The detailed neurological examination findings, Glasgow Coma Scale (GCS) scores, and vital signs were evaluated in all of the patients, and a 12-channel electrocardiogram (ECG) was performed. Those patients suspected of using SCs based on their medical histories and physical examinations underwent urine screenings.

Before the treatment with foley catheter for urine analysis. $5 \mathrm{cc}$ urine was collected from the patients with a history of SC use. The SC urine analyses were conducted using SC1,SC2,SC3 kits. (K1 (Synthetic Cannabinoids-1) urine enzyme immunoassay kit (LUCIO-Drug EIA SPICE (UR144/XLR-11) Ctalogue number: 320-00342EX), K2 (Synthetic Cannabinoids-2) urine enzyme immunoassay kit (Immunalysis Corporation, Pomona, CA, USA; catalogue number: 346-0025EX) and Synthetic Cannabinoids-3 urine enzyme immunoassay kit (Immuno-analysis Corporation, USA; catalogue number: 350-0025EX)). A total of 67 synthetic subunits (especially the JWH subgroups) were used in all kits. All of the patients in both groups underwent complete blood count, blood chemistry panel (glucose, sodium, potassium, urea, creatinine, alanine transaminase and aspartate transaminase) (ARCHITECT c16000, Abbott, Germany; catalogue number: 306941/R05-306954/R04), arterial blood gas and cardiac troponin I analyses (Siemens ADVIA Centaur CP immunoassay system, USA; Reference Number: 02790309).

After undergoing a bedside TCD USG examination, each patient was provided with symptomatic treatment according to his or her clinical situation. After treatment, all of the clinical outcomes, such as hospitalisation, intensive care and discharge, were recorded.

All of the TCD USG examinations were performed while the patient was laying on a bed in the supine position by a single interventional neurologist with 10 years of experience using a TCD USG device (MDT-2036; DWL, Germany). The TCD USG examination was performed using a $2 \mathrm{MHz}$ frequency ultrasound probe. The measurements were taken from the temporal window when the participants were in a supine position. The peak systolic flow rate, mean flow rate, pulsatility index $(\mathrm{PI})$ and resistivity index $(\mathrm{RI})$ of the right and the left anterior carotid arteries (ACAs), middle cerebral arteries (MCAs) and posterior cerebral arteries (PCAs) were measured from the temporal window before the treatment (Figure 1). ${ }^{9}$

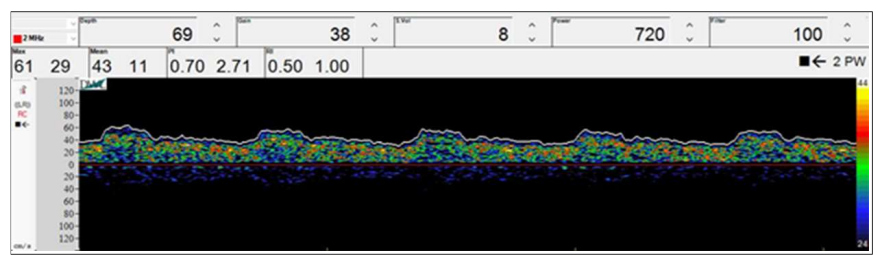

Figure 1: An example of a transcranial Doppler ultrasound of the target vessel after synthetic cannabinoid use (flow analysis in right middle cerebral artery).

The data were evaluated using IBM SPSS Statistics for Windows (Version 21.0; IBM Corp., Armonk, NY, USA). The sociodemographic and clinical characteristics of the patients were given as the mean \pm standard deviation, $95 \%$ confidence interval $(\mathrm{Cl})$ and percentage. Normal or abnormal distribution of values was tested by means of the Kolmogorov-Smirnov test, and the values for asymmetry and kurtosis between -2 and +2 were considered acceptable to prove normal distribution. ${ }^{10}$ Student's t-test was used for the comparison of the continuous variables, and the Chi-square test was used for the comparison of the intermittent variables. When appropriate, the $95 \%$ Cls were also calculated, and a p-value of less than 0.05 was considered to be statistically significant.

\section{RESULTS}

A total of 54 patients presented to the emergency medicine clinic after SC use during the study period. Sixteen 
Table I: Demographic characteristics and vital signs of the study groups.

\begin{tabular}{|c|c|c|c|c|}
\hline & All study groups & Cannabinoid + & Control group & $\mathrm{p}$-value \\
\hline Age (years), m ( $\pm S D)$ & $29.6( \pm 9.1)$ & $30.5( \pm 10.6)$ & $28.7( \pm 7.2)$ & 0.377 \\
\hline \multicolumn{5}{|l|}{ Gender } \\
\hline Male [n (\%)] & $68(91.9 \%)$ & $35(92.1 \%)$ & $33(91.6 \%)$ & 0.945 \\
\hline Female [n (\%)] & $6(8,1 \%)$ & $3(7,9 \%)$ & $3(8,3 \%)$ & \\
\hline Pulse rate (per minute), m ( $\pm S D)$ & $100.3( \pm 27.1)$ & $115.1( \pm 28.9)$ & $84.7( \pm 12.4)$ & $<0.001$ \\
\hline MAP $(\mathrm{mmHg}), \mathrm{m}( \pm \mathrm{SD})$ & $85.3( \pm 13.9)$ & $81.8( \pm 16.9)$ & $89.07( \pm 8.4)$ & 0.024 \\
\hline Temperature $\left({ }^{\circ} \mathrm{C}\right), \mathrm{m}( \pm \mathrm{SD})$ & $37( \pm 0.6)$ & $37.22( \pm 0.5)$ & $36.75( \pm 0.4)$ & $<0.001$ \\
\hline SpO2 (\%), m ( \pm SD) & $98.3( \pm 1.7)$ & $97.3( \pm 1.9)$ & $99.3( \pm 0.7)$ & $<0.001$ \\
\hline Respiratory rate (per minute), m ( $\pm S D$ ) & $16.9( \pm 6.5)$ & $20.4( \pm 7.3)$ & $13.2( \pm 1.9)$ & $<0.001$ \\
\hline
\end{tabular}

SD: Standard deviation, m: Mean, M: Males, F: Females $n$ : Number, MAP: Mean arterial pressure, $\mathrm{SpO}_{2}:$ Peripheral capillary oxygen saturation.

Table II: Basal laboratory findings of the study groups.

\begin{tabular}{|c|c|c|c|c|}
\hline & All study groups & Cannabinoid + & Control group & $\mathrm{p}$-value \\
\hline WBC m ( $\pm S D)$ & $10123( \pm 3628.4)$ & $11897( \pm 3812.8)$ & $8312( \pm 2302.7)$ & $<0.001$ \\
\hline Haemoglobin $\mathrm{m}( \pm S D)$ & $14.1( \pm 1.3)$ & $14.4( \pm 1.2)$ & $13.7( \pm 1.3)$ & 0.014 \\
\hline Platelets m ( $\pm S D)$ & $208.945( \pm 64.978,8)$ & $190.026( \pm 65.269,7)$ & $228.916( \pm 59.154,2)$ & 0.009 \\
\hline Urea $\mathrm{m}( \pm \mathrm{SD})$ & $30.8( \pm 7.3)$ & $32.9( \pm 7.1)$ & $28.6( \pm 6.9)$ & 0.009 \\
\hline Creatinine $\mathrm{m}( \pm \mathrm{SD})$ & $0.91( \pm 0.8)$ & $0.98( \pm 0.3)$ & $0.85( \pm 0.1)$ & 0.009 \\
\hline AST m ( $\pm S D)$ & $18( \pm 6.1)$ & $19.2( \pm 7)$ & $16.8( \pm 4.9)$ & 0.089 \\
\hline ALT m ( $\pm S D)$ & $23( \pm 5.8)$ & $22.8( \pm 5.8)$ & $23.3( \pm 5.7)$ & 0.748 \\
\hline Troponin I m ( $\pm S D)$ & $0.05( \pm 0.01)$ & $0.09( \pm 0.16)$ & $0.01( \pm 0.03)$ & 0.004 \\
\hline $\mathrm{pH} \mathrm{m} \mathrm{( \pm SD)}$ & $7.34( \pm 0.08)$ & $7.29( \pm 0.09)$ & $7.39( \pm 0.03)$ & $<0.001$ \\
\hline $\mathrm{PCO}_{2} \mathrm{~m}( \pm \mathrm{SD})$ & $47.48( \pm 10.9)$ & $55.09( \pm 9.47)$ & $39.44( \pm 5.13)$ & $<0.001$ \\
\hline $\mathrm{HCO}_{3} \mathrm{~m}( \pm \mathrm{SD})$ & $24.92( \pm 3.59)$ & $25.54( \pm 4.35)$ & $24.27( \pm 2.43)$ & 0.128 \\
\hline Lactate $\mathrm{m}( \pm \mathrm{SD})$ & $2.38( \pm 2.62)$ & $3.69( \pm 3.09)$ & $0.99( \pm 0.61)$ & $<0.001$ \\
\hline
\end{tabular}

SD: standard deviation, m: mean, WBC: white blood cell, AST:aspartate transaminase, ALT:alanine transaminase, PCO2: partial pressure of carbon dioxide, HCO3: bicarbonate.

Table III: Cerebral haemodynamics of the study groups via transcranial Doppler ultrasound measurements.

\begin{tabular}{|c|c|c|c|c|}
\hline & Cannabinoid + & Control group & $95 \% \mathrm{Cl}$ & $\overline{p \text {-value }}$ \\
\hline \multicolumn{5}{|l|}{ R-MCA } \\
\hline Peak m ( $\pm S D)$ & $68.92( \pm 16.68)$ & $90.92( \pm 14.76)$ & {$[(-29.31)-(-14.68)]$} & $<0.001$ \\
\hline Average $\mathrm{m}( \pm \mathrm{SD})$ & $45.05( \pm 13.21)$ & $58.03( \pm 11.44)$ & {$[(-18.72)-(-7.23)]$} & $<0.001$ \\
\hline $\mathrm{PI} \mathrm{m}( \pm \mathrm{SD})$ & $1.04( \pm 0.28)$ & $0.9( \pm 0.19)$ & {$[(0.03)-(0.25)]$} & 0.013 \\
\hline $\mathrm{RI}$ m ( $\pm S D)$ & $0.71( \pm 0.18)$ & $0.63( \pm 0.21)$ & {$[(0.01)-(0.17)]$} & 0.079 \\
\hline \multicolumn{5}{|l|}{ R-ACA } \\
\hline Peak m ( $\pm S D)$ & $61.34( \pm 16.47)$ & $86.39( \pm 16.85)$ & {$[(-32.77)-(-17.32)]$} & $<0.001$ \\
\hline Average $m( \pm S D)$ & $36.24( \pm 11.12)$ & $53.67( \pm 13.14)$ & {$[(-23.06)-(-11.8)]$} & $<0.001$ \\
\hline $\mathrm{PI} m( \pm \mathrm{SD})$ & $1.14( \pm 0.30)$ & $0.96( \pm 0.25)$ & {$[(0.06)-(0.32)]$} & 0.005 \\
\hline $\mathrm{RI} \mathrm{m}( \pm \mathrm{SD})$ & $0.74( \pm 0.24)$ & $0.58( \pm 0.09)$ & {$[(0.07)-(0.24)]$} & $<0.001$ \\
\hline \multicolumn{5}{|l|}{ R-PCA } \\
\hline Peak m ( $\pm S D)$ & $48.47( \pm 12.94)$ & $64.92( \pm 26.12)$ & {$[(-25.92)-(-7)]$} & 0.001 \\
\hline Average $\mathrm{m}( \pm \mathrm{SD})$ & $31.61( \pm 8.13)$ & $45.36( \pm 25)$ & {$[(22.28)-(-5.24)]$} & 0.002 \\
\hline $\mathrm{PI} \mathrm{m}( \pm \mathrm{SD})$ & $1.16( \pm 0.28)$ & $0.98( \pm 0.35)$ & {$[(0.04)-(0.33)]$} & 0.015 \\
\hline $\mathrm{RI}$ m(土SD) & $0.78( \pm 0.26)$ & $0.59( \pm 0.14)$ & {$[(0.09)-(0.29)]$} & $<0.001$ \\
\hline \multicolumn{5}{|l|}{ L-MCA } \\
\hline Peak m $( \pm S D)$ & $75.34( \pm 32.03)$ & $105.64( \pm 18.9)$ & {$[(-43.15)-(-17.44)]$} & $<0.001$ \\
\hline Average $m( \pm S D)$ & $51.13( \pm 21.87)$ & $73.28( \pm 13.17)$ & {$[(30.57)-(-13.72)]$} & $<0.001$ \\
\hline $\mathrm{PI} \mathrm{m}( \pm \mathrm{SD})$ & $1.04( \pm 0.34)$ & $0.83( \pm 0.17)$ & {$[(0.08)-(0.33)]$} & 0.002 \\
\hline $\mathrm{RI} \mathrm{m}( \pm \mathrm{SD})$ & $0.7( \pm 0.24)$ & $0.55( \pm 0.11)$ & {$[(0.06)-(0.24)]$} & 0.001 \\
\hline \multicolumn{5}{|l|}{ L-ACA } \\
\hline Peak m $( \pm S D)$ & $63.03( \pm 25.46)$ & $78.36( \pm 15.39)$ & {$[(-25.15)-(5.52)]$} & 0.003 \\
\hline Average $\mathrm{m}( \pm \mathrm{SD})$ & $38.63( \pm 14.77)$ & $52.81( \pm 13.35)$ & {$[(-20.5)-(-7.85)]$} & $<0.001$ \\
\hline $\mathrm{PI} m( \pm S D)$ & $1.08( \pm 0.46)$ & $0.84( \pm 0.2)$ & {$[(0.08)-(0.41)]$} & 0.004 \\
\hline $\mathrm{RI}$ m ( $\pm \mathrm{SD})$ & $0.69( \pm 0.25)$ & $0.55( \pm 0.09)$ & {$[(0.05)-(0.22)]$} & 0.003 \\
\hline \multicolumn{5}{|l|}{ L-PCA } \\
\hline Peak m ( $\pm S D)$ & $47.10( \pm 15)$ & $72.14( \pm 27.93)$ & {$[(35.35)-(-14.71)]$} & $<0.001$ \\
\hline Average $\mathrm{m}( \pm \mathrm{SD})$ & $29.84( \pm 10.4)$ & $50.14( \pm 25.27)$ & {$[(29.17)-(-11.43)]$} & $<0.001$ \\
\hline $\mathrm{PI}$ m ( $\pm S D)$ & $1.15( \pm 0.31)$ & $0.98( \pm 0.44)$ & {$[(0.01)-(0.35)]$} & 0.049 \\
\hline RI m ( $\pm S D)$ & $0.75( \pm 0.27)$ & $0.57( \pm 0.16)$ & {$[(0.08)-(0.29)]$} & 0.001 \\
\hline
\end{tabular}

Cl: Confidence interval, SD: Standard deviation, R: Right, L: Left, MCA: Middle cerebral artery, ACA: Anterior carotid artery, PCA: Posterior cerebral artery, PI: Pulsatility index, RI: Resistivity index. 
$(29.7 \%)$ of these patients were not included in the study because they did not qualify for inclusion. In total, 38 $(70.3 \%)$ patients who used SCs were included in this study. Thirty-six volunteers were included in the control group, with a total of 74 participants included in the study. The mean age of all of the participants was 29.6 $( \pm 9.1)$; $[30.5( \pm 10.6)$ in SC group, $28.7( \pm 7.2)$ in the control group]. Sixty-eight of the patients (91.9\%) were males. The demographic data and the vital signs of the patients at the time of admission have been summarised in Table I.

When the complaints at the time of admission were evaluated in the patients using SCs, the most common complaint was a loss of consciousness with the number of 27 patients $(71 \%)$, followed by nausea and vomiting $(n=20,52.6 \%)$, palpitations $(n=18,47.3 \%)$, dizziness $(n=13,34.2 \%)$, impairment of perception $(n=13,34.2 \%)$, anxiety disorder $(n=12,31.5 \%)$, chest pain $(n=7,18.4 \%)$, seizure $(n=7,18.4 \%)$, and dyspnoea $(n=5,13.2 \%)$. According to the physical examination findings of the patients, dysarthria in $39.5 \%(n=15)$, ataxia in $39.5 \%$ $(n=15)$, nystagmus in $28.9 \% \quad(n=11)$ [26.3\% $\quad(n=10)$ horizontal, $2.6 \%(n=1)$ rotatory], dysdiadochokinesis in $26.3 \%(n=10)$, and dysmetria in $15.8 \%(n=6)$ were detected.

The mean patient GCS score upon admission was 14.6 (min: 6, max: 15). According to the ECG results, sinus tachycardia $(n=24,63.1 \%)$ was the most common finding in the patients using SCs. The basal laboratory findings of the whole study group are shown in Table II.

In the synthetic cannabinoids group, statistically significant decreases in the cerebral blood flow peak systolic and mean average values, as well as increases in the pulsatility index and resistivity index were found when compared to the control group. The TCD USG findings in the study group are summarised in Table III.

The mean follow-up duration of the SC patients in the ED was $312.6( \pm 107.2)$ minutes. When the final status of the SC patients were examined, it was observed that 29 patients $(76.3 \%)$ were discharged from the ED, 4 patients $(10.5 \%)$ were admitted to the inpatient wards and 5 patients $(13.1 \%)$ were admitted to the intensive care unit.

\section{DISCUSSION}

Although SCs were first synthesised 20 years ago for medical purposes, their use has been increasing within different subgroups. They constitute an important public health issue. Cannabis affects almost every system of the body, particularly the central nervous system, cardiovascular, respiratuary and immune systems. ${ }^{11}$

Central nervous system findings and related symptoms have been reported predominantly in case-control series, but the level of evidence is limited due to the small size of the prospective studies. In this study, the cerebral blood flow changes due to SC use, which is increasing in prevalence (especially in the younger population), were examined. Our results showed statistically significant decreases in the cerebral blood flow peak systolic and mean values when compared to the control group, as well as increases in the PI and RI values. These changes caused intracranial vasospasms related to distal vasospasms.

There are a wide range of acute physiological intoxication symptoms related to SC use. The most common symptoms were palpitations, agitation, dizziness, nausea, vomiting and central nervous system symptoms, respectively. 12 Neurological and cardio-vascular side effects observed after cannabinoid poisoning generally respond to conventional supportive care, but severe outcomes may occur in a minority of cases, mainly observed with SCs. ${ }^{13}$ Orthostatic hypotension, followed by syncope when standing up, has been reported as the most common finding in marijuana users. Similarly, tachycardia, hypotension, dizziness and syncope were the most common findings in this study. When hypotension and tachycardia develop, cerebral arteries expand to maintain cerebral blood flow. Dizziness, followed by syncope, occurs when autoregulation mechanisms prove to be insufficient.

In the animal experimental study, conducted by Graham et al. ${ }^{14}$, after the injection of cannabinoid extracts, the blood pressure and respiratory rate decreased in a similar way, and it was suggested that the haemodynamic changes occurred to decrease the cerebral blood flow.

Although many conventional imaging modalities can be used to detect changes in the cerebral blood flow, TCD USG, which was first used in 1982 but currently has become widely used, enables an evaluation of the haemodynamic parameters at the bedside, such as the flow rate of the intracranial arteries in unstable patients, both noninvasively and without radiation. Moreover, cerebral autoregulation and vasoconstriction, partial obstruction and/or vasodilatation can be seen as a result of the changes in the blood flow rate in the large intracranial vessels as detected by TCD USG. In this study, the cerebral blood flow peak and mean flow rates decreased when compared to the control group in both the right and left ACAs, MCA and PCA, while the PI and $\mathrm{RI}$ values increased. Reductions in the blood flow rate and mean values lead to the dilatation of the arteries. Previous studies have shown increased intracranial pressure and decreased cerebral perfusion pressure leading to characteristic changes in TCD waveformsdecrease in diastolic velocity and increased in PI. 15

Thus, the changes in the cerebral resistance of small cortical blood vessels, the blood flow rate values 
recorded in large arteries, and the increases in the $\mathrm{PI}$ measurements show increases in the cerebral resistance in smaller veins away from the recording region. These changes can be associated with neurological symptoms in patients using SCs.

Regardless of the cause, the discontinuation of the cerebral blood flow results in a decrease in brain nutrition leading to ischemic cell death. ${ }^{16}$ In most stroke cases, the reduction in the cerebral blood flow is temporary, and the cell death does not occur immediately. There are several features of the endocannabinoid signalling system (ECS) that support its hypothesised involvement during ischemic injury. First, endocannabinoids and related lipids accumulate in ischemic tissues and support the hypothesis that the ECS is activated during ischemia. Second, the accumulated endocannabinoids support the general role of the ECS in the maintenance of metabolic homeostasis, and they support the sensitivity of the brain against stress. ${ }^{17}$ The CB1 cannabinoid receptor is found in the cerebral vascular bed, and its activation has been shown to cause vasodilatation. ${ }^{18}$ Importantly, this suggests that the activation of cerebrovascular CB1 receptors leads to a loss of autoregulation and exacerbates the effects of ischemia. ${ }^{19}$ There are many case studies indicating that SC use is associated with ischemic or haemorrhagic strokes, which support this thesis. 20,21 In a study by Wolf et al.,22 it was emphasised that the use of SCs may be a potential triggering factor for tertiary intracranial vasoconstriction, a pathology manifested by cerebral infarcts in up to $39 \%$ of the cases. As a result, vasoconstriction can be a mechanism that causes neuronal death through ischemia, with a significant reduction in the cerebral blood flow in the related regions.

The fact is that this study was single-centred and the regional factors limited the projection of the results to the general population. The consumption of a subgroup that was possibly not studied in the laboratory analysis, despite the presence of a history of SC use in some of the patients, and the exclusion of patients who consumed additional substances or ethanol, limited the number of cases. PCO2 levels were significantly higher in the SC group than in the volunteer group. The elevation of $\mathrm{PCO} 2$ may cause a decrease in cerebral blood flow. The authors think that the failure to differentiate changes in cerebral blood flow due to $S C$ or PCO2 elevation may be a limitation. Finally, TCD USG is user-dependent, and the fact that this was not controlled by other clinicians, may also be a limitation.

\section{CONCLUSION}

The results of this study showed that the symptoms of SC toxicity are variable, and they include a range of physical and psychological issues that may lead to serious complications. The cerebral blood flow and resistance changes due to SC use play roles in the main pathogenesis of the neurological symptoms, increasing the incidence of ischemic or haemorrhagic strokes, especially with chronic use. Due to the variable symptoms of SC toxicity, clinicians working in emergency services should consider SC toxicity when evaluating young adult patients presenting with unexplained agitation or neurological symptoms.

\section{ETHICAL APPROVAL:}

Approval for this study was obtained from the local Ethics Committee (KU GOKAEK, 2016/317).

\section{PATIENTS' CONSENT:}

All of the participants were informed before being included in the study, and consents were obtained from all the participants or their relatives.

\section{CONFLICT OF INTEREST:}

Authors declared no conflict of interest.

\section{AUTHORS' CONTRIBUTION:}

OK: Data collection, drafting, analysis and interpretation of data and manuscript writing.

UU: Initial design of the study, supervised the study, TCD ultrasound measurement,

SY: Literature search, proofreading of the manuscript, made substantial contributions to conception and design of the work.

NU: Contributed to initial concept design of the study, , supervised the study.

\section{REFERENCES}

1. Seely KA, Lapoint J, Moran JH, Fattore L. Spice drugs are more than harmless herbal blends: A review of the pharmacology and toxicology of synthetic cannabinoids. Prog Neuropsychopharmacol Biol Psychiatry 2012; 39:234-43.

2. Drummer $\mathrm{OH}$, Gerostamoulos D, Woodford NW. Cannabis as a cause of death: A review. Forensic Sci Int 2019; 298:298-306.

3. Musshoff F, Madea B, Kernbach-Wighton G, Bicker W, Kneisel S, Hutter $\mathrm{M}$, et al. Driving under the influence of synthetic cannabinoids ("Spice"): a case series. Int J Legal Med 2014; 128:59-64.

4. Lapoint J, James LP, Moran CL, Nelson LS, Hoffman RS, Moran JH. Severe toxicity following synthetic cannabinoid ingestion. Clin Toxicol 2011; 49:760-4.

5. Wolff V, Armspach JP, Lauer V, Rouyer O, Bataillard M, Marescaus $\mathrm{C}$, et al. Cannabis-related stroke: Myth or reality? Stroke 2013; 44:558-63.

6. Aydin G, Bakar B. Delayed intracerebral hemorrhage after synthetic cannabis (Bonsai) abuse; case report and literature review. Bull Emerg Trauma 2019; 7:330-4.

7. Richter JS, Quenardelle V, Rouyer O, Raul JS, Beaujeux R, Gény B. et al. A systematic review of the complex effects of cannabinoids on cerebral and peripheral circulation in animal models. Front Physiol 2018; 9:622. 
8. Sloan MA, Alexandrov AV, Tegeler $\mathrm{CH}$, Spencer MO, Caplan LR, Feldmann E, et al. Assessment: Transcranial doppler ultrasonography: Report of the therapeutics and technology assessment subcommittee of the American Academy of Neurology. Neurology 2004; 62:1468-81.

9. Bathala L, Mehndiratta MM, Sharma KV. Transcranial doppler: technique and common findings. Ann Indian Acad Neurol 2013; 16:174-9.

10. George D, Mallery P. SPSS for windows step by step: A simple guide and reference 17.0 update. Ed. $10^{\text {th }}$ Boston: Pearson; 2010.

11. Karam K, Abbasi S, Khan FA. Anaesthetic consideration in a cannabis addict. $J$ Coll Physicians Surg Pak 2015; 25(Suppl 1): S2-S3.

12. Courts J, Maskill V, Glue AGP. Signs and symptoms associated with synthetic cannabinoid toxicity: Systematic review. Australian Pyhchiatry 2016; 24:598-601.

13. Le Boisselier R., Alexandre J, Lelong-Boulouar V, Debruyne D. Focus on cannabinoids and synthetic cannabinoids. Clin Pharmacol Ther 2017; 101:220-9.

14. Graham JD, Li DM. Cardiovascular and respiratory effects of cannabis in cat and rat. Br J Pharmacol 1973; 49:1-10.

15. Minhas JS, Panerai RB, Ghaly G, Divall P, Robinson TG. Cerebral autoregulation in hemorrhagicstroke: A systematic review and meta-analysis of transcranial Doppler ultrasonography studies. J Clin Ultrasound 2019; 47:14-21.

16. Mehta SL, Manhas N, Raghubir R. Molecular targets in cerebral ischemia for developing novel therapeutics. Brain Res Rev 2007; 54:34-66.

17. Kunos G. Understanding metabolic homeostasis and imbalance: what is the role of the endocannabinoid system? Am J Med 2007; 120:S18-24.

18. Kolb B, Saber H, Fadel H, Rajah G. The endocannabinoid system and stroke: A focused review. Brain Circ 2019; 5:1?7.

19. Mathew RJ, Wilson WH, Davis R. Postural syncope after marijuana: A transcranial doppler study of the haemodynamics. Pharmacol Biochem Behav 2003; 75:309-18.

20. Rose DZ, Guerrero WR, Mokin MV, Gooch CL, Bozeman AC, Pearson JM, et al. Hemorrhagic stroke following use of the synthetic marijuana "spice". Neurology 2015; 85:1177-9.

21. El Mesbahy J, Chraa M, Louhab N, Kissani N. Hemorrhagic stroke after cannabis use in a young man. Rev Neurol 2017; 173:666-8.

22. Wolff V, Armspach JP, Lauer V, Rouyer O, Ducros A, Marescaux $\mathrm{C}$, et al. Ischaemic strokes with reversible vasoconstriction and without thunderclap headache: $\mathrm{A}$ variant of the reversible cerebral vasoconstriction syndrome? Cerebrovasc Dis 2015a; 39:31-8. 